\title{
The role of hepatic resection for gastric cancer liver metastases - literature review
}

\author{
Nicolae Bacalbasa1,2,3, Irina Balescu ${ }^{4}$, Mihaela Vilcu' ${ }^{2,3}$, Simona Dima1, Iulian Brezean ${ }^{2,3}$ \\ ${ }^{1}$ Center of Excellence in Translational Medicine, Fundeni Clinical Institute, Bucharest, Romania \\ 2"Carol Davila" University of Medicine and Pharmacy, Bucharest, Romania \\ 3"Ion Cantacuzino" Clinical Hospital, Bucharest, Romania \\ ${ }^{4}$ Ponderas Academic Hospital, Bucharest, Romania
}

\begin{abstract}
Gastric cancer remains one of the most aggressive malignancies which is frequently diagnosed in advanced stages of the disease. Therefore, the long term prognostic is extremely poor especially if surgery with curative intent is no longer feasible. When it comes to the predilection sites of metastatic disease with gastric origin, it seems that liver has a central role. The aim of this paper is to analyse the largest studies which investigated the potential benefit of gastric cancer liver metastases resection.
\end{abstract}

Keywords: gastric cancer, liver metastases, liver resection, survival

\section{INTRODUCTION}

Gastric cancer is one of the most commonly encountered malignancies worldwide and an important cause of cancer related death (1). Unfortunately, most cases are diagnosed in advanced stages of the disease, especially in countries in which screening tests for early detection is not implemented. In such cases the most commonly encountered patterns of spread are represented by peritoneal dissemination leading to the development of peritoneal carcinomatosis, lymphatic dissemination leading to the development of lymph node metastases and hematogenous dissemination leading to the apparition of parenchimatous metastases. Among cases which develop parenchimatous metastases, most often they occur via portal system and are located at the level of the liver parenchyma. However most often liver metastases develop as part of the systemic relapse; they are the sign of systemic disease and are associated with very poor prognostic. Development of hepato-bilio-pancreatic surgery leaded in the last decade to the permanent association of liver resections for hepatic metastases with different origins such as colorectal, ovarian and even breast cancer liver metastases (2). As for the patients with liver metastases from gastric cancer, the role of surgery is still under debate.

\section{THE ROLE OF SURGERY FOR} NON-COLORECTAL LIVER METASTASES

Initially the role of surgery for gastric cancer liver metastases has been investigated in studies which were focused on the issue of hepatic resection for non-colorectal liver metastases; therefore, in the study conducted by Slotta et al., 14 patients with metachronous liver metastases were included (3). Among these cases, the author reported a median overall survival of 17.3 months after liver resection with curative intent, significantly higher when compared to data reported so far for patients submitted to systemic chemotherapy (3). In a similar study conducted by Maeda et al. on 59 patients submitted to non-colorectal liver metastases resection, the authors reported 19 cases with primary gastric cancer; among the entire cohort there were eight cases who survived more than 5 years after liver 
resection, two of them originating from cases initially diagnosed with gastric cancer; moreover, the authors reported that five of the 59 patients had been initially diagnosed with unresectable disease. However after administration of neoadjuvant chemotherapy, five patients were converted to resectable disease, two of them presenting gastric primaries (4). In consequence, although patients with gastric cancer liver metastases were thought to have poorer outcomes after liver surgery when compared to other primaries such as colorectal or neuro-endocrine tumors, these outcomes seem to be significantly improved when compared to those reported after systemic chemotherapy as single strategy $(5,6)$.

Later on, after understanding the potential role of surgery, studies focused on this subtype of patients have been developed. Initially, due to the low number of cases submitted to this type of treatment, patients with synchronous and metachronous liver metastases were analysed together. One of the first studies conducted on this issue was conducted by Thelen et al. and was published in 2008 (7); in this study the authors included 24 patients submitted to surgery for synchronous (15 cases) and metachronous (9 cases) lesions with gastric cancer origin. Among cases submitted to surgery with curative intent the authors reported one year, three year and five year respectively overall survival of $53 \%, 22 \%$ and $15 \%$, the long term outcomes being significantly influenced by the completeness of resection. In the meantime, patients submitted to surgery for metachronous lesions seemed to have better outcomes when compared to cases submitted to surgery for synchronous lesion; moreover, the presence of extrahepatic disease seemed to have a negative influence of survival; however this fact did not reach statistically significant influence (7).

\section{THE ROLE OF SURGERY IN SYNCHRONOUS GASTRIC CANCER LIVER METASTASES}

When it comes to the concept of debulking surgery including liver resection for advanced stage gastric cancer with liver metastases, various opinions have been published so far; therefore, while certain authors consider that only metachronous lesions are susceptible for resection, other papers also take in consideration surgery for synchronous lesions (8-11).

Initially it has been considered that the presence of synchronous liver metastases from gastric cancer is the sign of a systemic disease and should be a formal contraindication for resection. Most often the role of surgery for synchronous gastric cancer liver metastases has been investigated in the absence of extrahepatic diseases; in the meantime, another essential condition in order to proceed for gastric cancer liver metastases resection is to achieve a macroscopic complete resection (8).

However, later on, studies have demonstrated that a significant benefit in terms of survival might be achieved if radical surgery is performed; therefore, in the study conducted by Qiu et al. the author reported a median survival of 38 months among the 25 patients submitted to radical surgery for gastric cancer with synchronous liver metastases (8). In the meantime the same study compared the postoperative outcomes of patients submitted to radical surgery for synchronous liver metastases to those of patients submitted to non-surgical procedures such as systemic chemotherapy, local ablation or hepatic arterial infusion and demonstrated that in the latter category the median overall survival was of only 7-13 months. Moreover, association of adjuvant chemotherapy to radical resection proved to be the most efficient therapeutic strategy in Qiu's study, in this particular subgroup of cases the overall survival being of 43 months (8).

One of the largest and most interesting studies published so far on the issue of surgical resection in synchronous gastric cancer liver metastases has been recently published by Picado et al. in the Journal of Surgical Research (12). The study included 3,175 patients diagnosed with synchronous gastric cancer liver metastases; among these cases 2,979 were treated with systemic chemotherapy while the remaining 196 cases were surgically treated. The long term outcomes were significantly better among cases submitted to surgery when compared to systemic chemotherapy (16 months versus 9.7 months, $p<0.001$ ); in the meantime among cases submitted to surgery, patients in whom neoadjuvant chemotherapy was associated also trend to have a better outcome when compared to those submitted to surgery followed by adjuvant chemotherapy (18.9 months versus 14.8 months, $p=0.011$ ) (12).

\section{THE ROLE OF SURGERY IN METACHRONOUS GASTRIC CANCER LIVER METASTASES}

Although surgery with curative intent for gastric cancer has been performed, a significant number of cases will develop systemic recurrence, liver being one of the most commonly involved sites of relapse. In a study conducted by Marrelli et al. on 
208 patients 28 of them developed liver relapse, this finding being statistically significant associated with the presence of lymph node involvement at the time of initial surgery, higher preoperative levels of serum markers such as CEA, CA19-9 and CA 72-4 as well as with the intestinal histotype (13).

The role of surgery for metachronous liver metastases from gastric cancer has been widely studied so far in both single center as well as in multicentric studies, promising results being reported.

An interesting study conducted on this issue was published in 2009 in the European Journal os Surgical Oncology by Tiberio et al. and included 73 patients diagnosed with gastric cancer liver metastases (14); among these cases there were 45 cases which were submitted to supportive care, 17 cases submitted to systemic chemotherapy and 11 cases submitted to surgery. the most important prognostic factors were identified to be the type of treatment at the time when metastatic disease was demonstrated as well as the biological characteristics of the primary tumor; therefore it seems that patients presenting advanced stage at diagnostic (defined by the $\mathrm{T}$ and $\mathrm{N}$ stages) as well as those presenting poorly differentiated tumors reported a poorer outcome even after surgery with curative intent for hepatic disease. However, it seems that the most powerful factor influencing the long term outcomes was represented by the type of treatment at the time of liver metastases diagnostic, surgery being significantly associated with improved survival when compared to the other strategies. Therefore, the authors recommended that surgery should be taken in consideration whenever possible in gastric cancer liver metastases in order to improve the chances for long term survival (14).

In the meantime, Petrelli's meta-analyses on the subject of metachronous liver metastases from gastric cancer conducted on 870 subjects demonstrated that surgery was associated with significantly better outcomes when compared to other conservative procedures (15).

\section{PROGNOSTIC FACTORS AFTER LIVER RESECTION FOR GASTRIC CANCER HEPATIC METASTASES}

In order to investigate which cases benefit most after liver resection for gastric cancer liver metastases Qiu et al. conducted a study on 25 cases submitted to liver resections synchronously with gastric cancer resection and classified the patients in survivors (if the overall survival was longer than three years after surgery) and non-survivors if the overall survival was shorter. According to this classification survivors were more likely to have a single metastatic lesion. Another prognostic factor which seemed to be significantly correlated with the long term outcomes was represented by the association of adjuvant chemotherapy (8).

Another factor which was analysed in multiple studies is represented by the dimension of the liver metastases; however conflicting results have been reported so far. Therefore while certain authors came to demonstrate that smaller lesions were associated with better overall prognostic (16), other papers failed to demonstrate any significant correlation between the two parameters (17-20).

Other factors which seem to influence the long term outcomes after liver resection for gastric cancer hepatic metastases are represented by advanced age at the time of diagnostic or treatment in non-academic institutions (12).

\section{THE ROLE OF RE-RESECTION FOR LIVER METASTASES FROM GASTRIC CANCER}

While in cases diagnosed with colo-rectal cancer the role of re-resection has been widely debated, in gastric cancer patients this strategy has been rarely taken in consideration. Although data reported so far is scarce, it seems that in selected cases with liver limited recurrent disease repeated hepatectomy might be an interesting option $(8,9,21)$. Therefore, in the study conducted by Qiu et al. two patients out of the 25 cases initially submitted to surgery for gastric cancer with synchronous liver metastases were also submitted to re-resection for liver limited recurrence and reported a survival benefit of 13 and 60 months respectively after re-resection (8).

\section{CONCLUSIONS}

Latest studies conducted on the theme of liver resection for gastric cancer hepatic metastases have shown promising results especially in cases with favourable cancer biology as well as in cases with hepatic confined disease in which radical resection with negative resection margins is achievable. However data is still scarce, larger studies being still needed.

\section{Acknowledgement}

This work was supported by the project entitled „Multidisciplinary Consortium for Supporting the 
Research Skills in Diagnosing, Treating and Identifying Predictive Factors of Malignant Gynecologic
Disorders", project number PN-III-P1-1.2-PCCDI2017-0833.

Conflict of interest: none declared

\section{REFERENCES}

1. Leung WK, Wu MS, Kakugawa et al. Screening for gastric cancer in Asia: Current evidence and practice. Lancet Oncol. 2008; 9: 279-287.

2. Choti MA, Sitzmann JV, Tiburi MF et al. Trends in long-term survival following liver resection for hepatic colorectal metastases. Ann.Surg. 2002; 235: 759-766.

3. Slotta J E, Schuld J, Distler S et al. Hepatic resection of non-colorectal and non-neuroendocrine liver metastases - survival benefit for patients with non-gastrointestinal primary cancers - a case-controlled study. Int.J.Surg. 2014; 12: 163-168.

4. Maeda $Y$, Shinohara T, Katayama T et al. Hepatectomy for liver metastases in non-colorectal, non-neuroendocrine cancer patients. The survival benefit in primary unresectable cases. Int.J.Surg. 2015; 22: $136-142$.

5. Kerkar SP, Kemp CD, Avital I. Liver resections in metastatic gastric cancer. HPB (Oxford) 2010; 12: 589-596.

6. Parisi A, Trastulli S, Ricci F et al. Analysis of long-term results after liver surgery for metastases from colorectal and non-colorectal tumors: A retrospective cohort study. Int.J.Surg. 2016; 30: 25-30.

7. Thelen A, Jonas $\mathrm{S}$, Benckert $\mathrm{C}$ et al. Liver resection for metastatic gastric cancer. Eur.J.Surg.Oncol. 2008; 34: 1328-1334.

8. Qiu JL, Deng MG, Li W, Zou RH et al. Hepatic resection for synchronous hepatic metastasis from gastric cancer. Eur.J.Surg. Oncol. 2013; 39: 694-700.

9. Okano K, Maeba T, Ishimura K et al. Hepatic resection for metastatic tumors from gastric cancer. Ann.Surg. 2002; 235: 86-91.

10. Ambiru S, Miyazaki M, Ito $\mathrm{H}$ et al. Benefits and limits of hepatic resection for gastric metastases. Am.J.Surg. 2001; 181: 279-283.

11. Schildberg CW, Croner R, Merkel S et al. Outcome of operative therapy of hepatic metastatic stomach carcinoma: a retrospective analysis. World J.Surg. 2012; 36: 872-878.

12. Picado O, Dygert L, Macedo FI et al. The Role of Surgical Resection for Stage IV Gastric Cancer With Synchronous Hepatic Metastasis. J.Surg.Res. 2018; 232: 422-429.
13. Marrelli D, Roviello F, De Stefano A et al. Risk factors for liver metastases after curative surgical procedures for gastric cancer: A prospective study of 208 patients treated with surgical resection. J.Am.Coll.Surg. 2004; 198: 51-58.

14. Tiberio G A, Coniglio A, Marchet A et al. Metachronous hepatic metastases from gastric carcinoma: A multicentric survey. Eur.J.Surg. Oncol. 2009; 35: 486-491.

15. Petrelli F, Coinu A, Cabiddu M et al. Hepatic resection for gastric cancer liver metastases: A systematic review and meta-analysis. J.Surg. Oncol. 2015; 111: 1021-1027.

16. Sakamoto Y, Sano T, Shimada K et al. Favorable indications for hepatectomy in patients with liver metastasis from gastric cancer. J.Surg. Oncol. 2007; 95: 534-539.

17. Koga R, Yamamoto J, Ohyama S et al. Liver resection for metastatic gastric cancer: experience with 42 patients including eight long-term survivors. Jpn.J.Clin. Oncol. 2007; 37: 836-842.

18. Ueda K, Iwahashi M, Nakamori M et al. Analysis of the prognostic factors and evaluation of surgical treatment for synchronous liver metastases from gastric cancer. Langenbecks Arch.Surg. 2009; 394: 647-653.

19. Tsujimoto $\mathrm{H}$, Ichikura $\mathrm{T}$, Ono $\mathrm{S}$ et al. Outcomes for patients following hepatic resection of metastatic tumors from gastric cancer. Hepatol. Int. 2010; 4: 406-413.

20. Zacherl J, Zacherl M, Scheuba C et al. Analysis of hepatic resection of metastasis originating from gastric adenocarcinoma. J.Gastrointest. Surg. 2002; 6: 682-689.

21. Sakamoto $\mathrm{Y}$, Ohyama S, Yamamoto J et al. Surgical resection of liver metastases of gastric cancer: An analysis of a 17-year experience with 22 patients. Surgery 2003; 133: 507-511. 